\title{
A Decentralized Fault Accommodation Scheme For Nonlinear Interconnected Systems
}

\author{
H. Ferdowsi and S. Jagannathan \\ Department of Electrical \& Computer Engineering, \\ Missouri University of Science and Technology, Rolla, MO, USA \\ E-mail: hfqk6@mst.edu
}

\begin{abstract}
In this paper, a novel decentralized detection and accommodation (FDA) methodology is proposed for interconnected nonlinear continuous-time systems by using local subsystem states alone in contrast with traditional distributed FDA schemes where the entire measured or the estimated state vector is needed. First, the detection scheme is revisited where a network of local fault detectors (LFD) is proposed. A fault is detected by generating a residual from the measured and estimated state vectors locally and the fault dynamics are estimated by using an online approximator upon detection. Subsequently, a fault accommodation scheme is initiated in the subsystem by using a second online approximator to augment the control input of each subsystem in order to minimize the effects of the faults on the overall system. Decentralization avoids the transmission of the entire system state vector to each subsystem. Finally the proposed methods are verified in the simulation environment.
\end{abstract}

\section{INTRODUCTION}

Many industrial systems for example power or water distribution networks, telecommunication networks, and so on are complex, large-scale, spatially distributed and interconnected nonlinear systems. Suitable fault detection schemes are required for these systems to ensure that a fault at any given location can be detected at an incipient stage in order to prevent catastrophic failure of the overall system.

Based on the growth rate, faults are classified into abrupt and incipient categories. When an abrupt fault occurs, it is imperative to detect the fault and shut down the system as soon as possible to replace or repair the faulty component. But in case of incipient faults, the system can keep running for a possibly long period of time without any serious risk of damage to the whole system. However, in such circumstances, a fault accommodation scheme is required to enable the system to continue its intended operation without significant performance degradation. In other words, fault accommodation allows the system to keep working with the desired performance after occurrence of an incipient fault, thus increasing the reliability and reducing the down time.

In order to perform fault accommodation, the fault needs to be detected first. Basically two types of fault detection schemes are available in the literature [1]: data-driven and model-based schemes. Model-based schemes are more desirable as they function online in the absence of faulty or healthy data. Model-based schemes use a state estimator as a reference model for fault detection by using high gain observers [2], neural network observers [3], and geometric techniques [4]. On the other hand, centralized fault accommodation is also available by utilizing observer-based schemes [5], adaptive estimators [6], and so on.

Recently design of distributed fault detection schemes for interconnected systems have been proposed by using $H_{\infty}$ based method [7], multiblock kernel partial least squares [8], fuzzy observers [9] and so on. In addition, several distributed accommodation schemes [10-12] have been introduced. However, most of them [7-12] require either entire state or estimated state vector for each local fault detector (LFD) since these are merely distributed schemes. Typically, it is not always possible to provide the information of the entire state or its estimated value for LFDs of a large-scale spatially distributed system unless some information from other subsystems is communicated. Even if this is possible, the information will be delayed and outdated, and expensive.

In contrast to the aforementioned schemes [7-12], in this paper our objective is to design a LFD for each subsystem. Each LFD is a nonlinear fault detection observer which uses local states of that subsystem alone to detect a fault and to approximate its dynamics. Upon detection, a fault accommodation framework is proposed in which the control input of each subsystem is augmented in order to accommodate the effects of the fault by using the local subsystem states alone.

The output of the fault detection OLA will continue to be utilized in the faulty subsystem to mitigate the local fault dynamics. A second OLA is also initiated in the faulty subsystem for accommodation once the location of the fault to a subsystem is identified.

Since a fault in one subsystem can propagate to other subsystems via interconnection terms, an accommodation OLA will be initiated in each of the other subsystems to mitigate this effect. Here, utilization of two different OLAs 
for detection and accommodation enables the proposed scheme to provide an estimation of fault dynamics and perform accommodation simultaneously.

Thus this paper introduces a truly decentralized fault detection and accommodation scheme for interconnected nonlinear continuous-time systems. Next, the interconnected nonlinear system description is presented followed by decentralized detection and accommodation schemes.

\section{SYSTEM DESCRIPTION}

Consider a nonlinear continuous-time system that is comprised of $\mathrm{N}$ interconnected subsystems. The dynamics of the $\mathrm{i}^{\text {th }}$ subsystem with $n_{i}$ states are described by

$$
\begin{gathered}
\dot{x}_{i j}(t)=x_{i(j+1)}(t) \quad j=1,2, \ldots, n_{i}-1 \\
\dot{x}_{i n_{i}}(t)=f_{i}\left(x_{i}(t), u_{i}(t)\right)+g_{i}\left(x_{i}(t), \bar{x}_{i}(t)\right) \\
+\eta_{i}(x(t))+\Omega_{i}\left(t-t_{0}\right) h_{i}\left(x_{i}(t)\right)
\end{gathered}
$$

where $u_{i} \in \mathbb{R}$ is the local control input, $x_{i} \in \mathbb{R}^{n_{i}}$ is the local state vector, $\bar{x}_{i} \in \mathbb{R}^{\bar{n}_{i}}$ is the vector of interconnection states, $f_{i}: \mathbb{R}^{n_{i}} \times \mathbb{R} \rightarrow \mathbb{R}^{n_{i}}$ and $g_{i}: \mathbb{R}^{n_{i}} \times \mathbb{R}^{\bar{n}_{i}} \rightarrow \mathbb{R}$ represent the known local and unknown interconnection functions respectively, $\eta_{i}: \mathbb{R}^{n_{i}} \rightarrow \mathbb{R}$ denote the system uncertainties, and $h_{i}: \mathbb{R}^{n_{i}} \rightarrow \mathbb{R}$ is the local fault function. Furthermore, it is assumed in this work that all the local states are measurable.

The time profile of a fault $\Omega_{\mathrm{i}}\left(t-t_{0}\right)$ is modeled by

$$
\Omega_{i}\left(t-t_{0}\right)= \begin{cases}0, & \text { if } t<t_{0} \\ 1-e^{-\bar{x}_{i}\left(t-t_{0}\right)}, & \text { if } t \geq t_{0}\end{cases}
$$

where $\bar{\kappa}_{i}$ is an unknown constant that represents the rate at which a fault occurs. A larger value of $\bar{\kappa}_{i}$ indicates that the fault is closer to an abrupt fault while small values of $\bar{\kappa}_{i}$ indicate that the fault is of an incipient type. The use of such time profiles is common in fault diagnosis literature [11]. It should be mentioned that the fault time profile does not necessarily have to be in exponential form, but it can have the form of any increasing function, whose value is zero before the time of fault occurrence.

There are many faults in the real world that can be modeled accordingly. Consider an axial piston pump as an example. This system can be described in state space form where each state will represent the pressure of one of the pistons. Pistons can have piston wear or slipper wear fault. These faults negatively affect the system's performance, and this effect keeps increasing as the wear keeps increasing over time. However, it might take a long time before the fault magnitude is large enough to be detected, because these faults are incipient and grow slowly.

Next two standard assumptions, which are needed, are presented.
Assumption 1: The modeling uncertainty is bounded, i.e. $\left\|\eta_{i}(x(t))\right\| \leq \eta_{i_{M}}, \forall(x, u) \in(\chi \times U), i=1,2, \ldots, N$, where $\eta_{i_{M}}$ is a known positive constant.

Remark 1: Assumption 1 is required to differentiate between faults and modeling uncertainties and to analytically define fault detection thresholds to prevent false or missed alarms.

Assumption 2: A fault occurs only in one of the subsystems and fault functions can be expressed as nonlinear in the unknown parameters (NLIP) [13]. Then, the fault functions can be approximated by using two-layer NNs with nonlinear activation functions.

Next the proposed fault detection scheme is introduced.

\section{DeCEnTRALized Fault Detection}

Our objective for the fault detection scheme is to design a network of LFDs such that a LFD monitors a subsystem using the local states of that subsystem. Now consider the nonlinear observer for the subsystem ' $\mathrm{i}$ ' described by

$$
\begin{aligned}
\dot{\hat{x}}_{i j}(t)=- & \lambda \hat{x}_{i j}(t)+x_{i(j+1)}(t)+\lambda x_{i j}(t) \quad j=1,2, \ldots, n_{i}-1 \\
\dot{\hat{x}}_{i n_{i}}(t)=- & \lambda \hat{x}_{i n_{i}}(t)+f_{i}\left(x_{i}(t), u_{i}(t)\right)+\lambda x_{i n_{i}}(t) \\
& +\hat{h}_{i}\left(x_{i}(t) ; \hat{\theta}_{i}(t)\right)
\end{aligned}
$$

where $\hat{x}_{i}(t) \in \mathbb{R}^{n_{i}}$ is the estimated local state vector of the $\mathrm{i}^{\text {th }}$ subsystem, $\hat{h}_{i}: \mathbb{R}^{n_{i}} \times \mathbb{R}^{p_{i}} \rightarrow \mathbb{R}$ is the output of the online approximator with $\hat{\theta}_{i} \in \mathbb{R}^{p_{i}}$ being the set of unknown parameters, and $\lambda$ is a user defined scalar. Initial values of the LFD are taken as $\hat{x}_{i}(0)=\hat{x}_{i_{0}}, \hat{\theta}_{i}(0)=\hat{\theta}_{i_{0}}$, such that $\hat{h}_{i}\left(x_{i}, \hat{\theta}_{i_{0}}\right)=0, \forall x_{i} \in X_{i}$.

Define a local detection residual $e_{i}=x_{i}-\hat{x}_{i}$. Under healthy system operation, the local residual dynamics are described by

$$
\begin{array}{lc}
\dot{e}_{i j}(t)=-\lambda e_{i j}(t) & j=1,2, \ldots, n_{i}-1 \\
\dot{e}_{i n_{i}}(t)=-\lambda e_{i n_{i}}(t)+g_{i}\left(x_{i}(t), \bar{x}_{i}(t)\right)+\eta_{i}(x(t))
\end{array}
$$

Since the system states are bounded in the healthy operation, the interconnection terms $g_{i}\left(x_{i}(t), \bar{x}_{i}(t)\right)$ are bounded $\left(g_{i}(x(t)) \leq g_{i_{M}}\right.$ in healthy conditions). The uncertainty term $\eta_{i}(x(t))$ is also bounded by Assumption 1 . So by appropriate selection of $\lambda$, the local detection residual will remain bounded prior to the occurrence of a fault.

In this work, neural networks (NNs) are used as online approximators, which are off prior to the detection of a fault. When the local detection residual of the $k^{\text {th }}$ subsystem exceeds its detection threshold, $\rho_{k}$, a fault is declared active in that particular subsystem, and the local OLA that generates $\hat{h}_{k}($.$) is initiated. Upon detection, unknown parameter vector$ of the $k^{\text {th }}$ OLA will be tuned using the following update law 


$$
\dot{\hat{\theta}}_{k}(t)=H\left[\left\|e_{k}(t)\right\|-\rho_{k}\right]\left(\alpha_{k} e_{k n_{k}}(t) \phi_{k}\left(x_{k}(t)\right)-\gamma_{k} \hat{\theta}_{k}(t)\right)
$$

where $\alpha_{k}>0$ is the $\mathrm{NN}$ learning rate, $0<\gamma_{k}<1$, $\phi_{k}\left(x_{k}(t)\right)$ is the basis function such as sigmoid or RBF, and $H(x)$ is the Heaviside operator defined by $H(x)=\left\{\begin{array}{ll}1 & \text { if } x \geq 0 \\ 0 & \text { if } x<0\end{array}\right.$. Then, the OLA output is given by $\hat{h}_{k}(t)=\hat{\theta}_{k}^{T}(t) \phi_{k}\left(x_{k}(t)\right)$.

Upon detection, the local detection residual dynamics of subsystem $k$ becomes

$$
\begin{aligned}
\dot{e}_{k j}(t)= & -\lambda e_{k j}(t) \quad j=1,2, \ldots, n_{k}-1 \\
\dot{e}_{k n_{k}}(t)= & -\lambda e_{k n_{k}}(t)+g_{k}\left(x_{k}(t), \bar{x}_{k}(t)\right)+\eta_{k}(x(t)) \\
& +h_{k}\left(x_{k}(t)\right)-\hat{h}_{k}\left(x_{k}(t) ; \hat{\theta}_{k}(t)\right)
\end{aligned}
$$

By asserting the NLIP assumption on the fault function to get

$$
\begin{aligned}
\dot{e}_{k n_{k}}(t)= & -\lambda e_{k n_{k}}(t)+g_{k}\left(x_{k}(t), \bar{x}_{k}(t)\right)+\eta_{k}(x(t)) \\
& +\tilde{\theta}_{k}^{T}(t) \phi_{k}\left(x_{k}(t)\right)+\varepsilon_{k}(t)
\end{aligned}
$$

where $\tilde{\theta}_{k}(t)=\theta_{k}-\hat{\theta}_{k}(t)$ is the parameter estimation error and $\varepsilon_{k}(t)$ is the OLA approximation error. Upon detection of the fault in subsystem ' $k$ ', other subsystems are notified and their fault detection OLADs are not tuned online.

Consequently, for the rest of the subsystems $\tilde{\theta}_{i}(t)=0$ and $\varepsilon_{i}(t)=0$, and their local residual dynamics are given by

$$
\begin{array}{r}
\dot{e}_{i i_{i}}(t)=-\lambda e_{i i_{i}}(t)+g_{i}\left(x_{i}(t), \bar{x}_{i}(t)\right)+\eta_{i}(x(t)) \\
\text { for } i=1, \ldots, N, i \neq k
\end{array}
$$

Next the following assumption on the interconnection terms is needed before the stability of the proposed observer is discussed upon detection.

Assumption 3: The interconnection terms are unknown and expressed as $\quad g_{i}(x(t)) \leq \xi_{i 0}+\sum_{j=1}^{N} \xi_{i j}\left(e_{j}(t)\right), i=$ $1,2, \ldots, N$, where $\xi_{i 0}$ for $i=1,2, \ldots, N$ and $\xi_{i j}($.$) for i, j=$ $1,2, \ldots, N$ are unknown constants and smooth functions respectively such that $\xi_{i j}(0)=0$.

Remark 2: Assumption 3 has been used in a variety of forms in the decentralized control or fault detection literature [14]. Although after the detection of faults, one of the residuals, namely $e_{k}$, can go to zero or near zero because of the approximation property of the OLA, other residuals do not, because OLAs in their subsystems are offline unless a fault is detected in the other subsystems. Therefore the bound on the interconnection terms defined in Assumption 3 will not decrease upon fault detection.

The stability of the proposed FD observer is revisited in the following theorem from [15]. A new approach is taken in the proof of this theorem which is based on combining individual Lyapunov functions for all the subsystems.

Theorem 1 (Fault Detection Observer Performance): Let the LFD observer network defined in (2) be used to monitor the overall system described by (1), with the local OLA being turned on upon the detection of a fault in the $k^{\text {th }}$ subsystem. Let the update law in (3) be used to update the unknown parameter vector $\hat{\theta}_{k}(t)$. In the presence of system uncertainties, the local FD residuals, $e_{i}(t) i=1, \ldots, N$, and the parameter estimation error, $\tilde{\theta}_{k}(t)$, will be uniformly ultimately bounded (UUB), provided the design parameters are selected as

$$
\lambda>\frac{3+N+\xi_{i_{\max }}^{2}}{2}
$$

So the local detection residuals and the parameter estimation errors of the online OLA are UUB.

Remark 3: The bound on $e_{k}$ is separated from all the other residuals because it is larger than the bound on other residuals. However the bounds on all the residuals, including $e_{k}$, can be combined by taking the supremum operator.

Remark 4: The fault location is identified by communicating the fault detection times of each subsystem to a centralized unit which then compares and finds the minimum time when the fault is detected and its associated subsystem. Then this subsystem will become the one where the fault is located.

\section{DeCENTRALIZEd Fault ACCOMmodation}

Upon determination of the fault location via fault isolation, fault accommodation is performed to mitigate the effect of the fault both in the faulty subsystems and in the other subsystems, because the fault in one subsystem can affect the others through the interconnection terms.This accommodation is performed in all the subsystems including the one where a fault is detected. As mentioned before, the subsystem where a fault occurs will have two OLAs one for approximating the fault dynamics while the other for accommodation whereas the other subsystems each will have one OLA for accommodation.

First we define a suitable control input in the healthy conditions, when the $\mathrm{i}^{\text {th }}$ subsystem dynamics are described by

$$
\begin{aligned}
& \dot{x}_{i j}(t)=x_{i(j+1)}(t) \quad j=1,2, \ldots, n_{i}-1 \\
& \dot{x}_{i i_{i}}(t)=f_{i}\left(x_{i}(t), u_{i}(t)\right)+g_{i}\left(x_{i}(t), \bar{x}_{i}(t)\right)+\eta_{i}(x(t))
\end{aligned}
$$

Using the technique introduced in [16] this can be rewritten as

$$
\begin{aligned}
\dot{x}_{i j}(t)= & x_{i(j+1)}(t) \quad j=1,2, \ldots, n_{i}-1 \\
\dot{x}_{i n_{i}}(t)= & f_{i 1}\left(x_{i}(t)\right)+f_{i 2}\left(x_{i}(t)\right) u_{i}(t) \\
& +g_{i}\left(x_{i}(t), \bar{x}_{i}(t)\right)+\eta_{i}(x(t))
\end{aligned}
$$


where $f_{i 1}($.$) and f_{i 2}($.$) are known smooth functions. If the$ control objective is to make $x_{i 1}(t)$ track the desired trajectory, $x_{i d}(t)$, the tracking error dynamics in healthy operating condition, is described by

$$
\begin{aligned}
\dot{\bar{e}}_{i j}(t)= & \bar{e}_{i(j+1)}(t) \quad j=1,2, \ldots, n_{i}-1 \\
\dot{\bar{e}}_{i n_{i}}(t) & \dot{x}_{i n_{i}}(t)-x_{i d}^{\left(n_{i}\right)}(t)=f_{i 1}\left(x_{i}(t)\right)+f_{i 2}\left(x_{i}(t)\right) u_{i}(t)+g_{i}\left(x_{i}(t), \bar{x}_{i}(t)\right) \\
& +\eta_{i}(x(t))-x_{i d}^{\left(n_{i}\right)}(t)
\end{aligned}
$$

where $\bar{e}_{i}$ represents the vector of local tracking errors in the $\mathrm{i}^{\text {th }}$ subsystem. Now let $A_{i}$ and $B_{i}$ be defined as

$$
A_{i}=\left[\begin{array}{ccccc}
0 & 1 & 0 & \ldots & 0 \\
0 & 0 & 1 & \ldots & 0 \\
\vdots & \vdots & \vdots & \ddots & \vdots \\
0 & 0 & 0 & \ldots & 1 \\
0 & 0 & 0 & \ldots & 0
\end{array}\right] \quad B_{i}=\left[\begin{array}{c}
0 \\
0 \\
\vdots \\
0 \\
1
\end{array}\right]
$$

Then tracking error dynamics can be rewritten in matrix form

$$
\begin{aligned}
\dot{\bar{e}}_{i}(t)=A_{i} \bar{e}_{i}(t)+B_{i}[ & f_{i 1}\left(x_{i}(t)\right)+f_{i 2}\left(x_{i}(t)\right) u_{i}(t) \\
& \left.+g_{i}\left(x_{i}(t), \bar{x}_{i}(t)\right)+\eta_{i}(x(t))-x_{i d}^{\left(n_{i}\right)}(t)\right]
\end{aligned}
$$

Since $g_{i}\left(x_{i}(t), \bar{x}_{i}(t)\right)$ and $\eta_{i}(x(t))$ are both bounded in healthy operating conditions, the control input $u_{i}(t)=$ $u_{i d}(t)$ defined by

$$
u_{i d}(t)=f_{i 2}\left(x_{i}(t)\right)^{-1}\left(x_{i d}^{\left(n_{i}\right)}(t)-K_{i}^{T} \bar{e}_{i}(t)-f_{i 1}\left(x_{i}(t)\right)\right)
$$

can keep tracking error bounded if $K_{i} \in \mathbb{R}^{n_{i}}$ is selected such that $A_{i}-B_{i} K_{i}^{T}$ is Hurwitz.

With the presence of fault, the control input $u_{i d}(t)$ can no longer satisfy the control objective. Thus after detection, the local control input is selected as $u_{i}(t)=u_{i d}(t)+u_{i c}(t)$, where $u_{i c}(t)$ is the augmented term to keep the local states track their desired trajectories after the fault. Using this augmented input, the tracking error dynamics can be represented as

$$
\begin{aligned}
\dot{\bar{e}}_{i}(t)=A_{i} \bar{e}_{i}(t)+B_{i}[ & -K_{i}^{T} \bar{e}_{i}(t)+f_{i 2}\left(x_{i}(t)\right) u_{i c}(t)+\eta_{i}(x(t)) \\
& \left.+g_{i}\left(x_{i}(t), \bar{x}_{i}(t)\right)+\Omega_{i}\left(t-t_{0}\right) h_{i}\left(x_{i}(t)\right)\right]
\end{aligned}
$$

Ideally, $u_{i c}(t)$ should be selected as

$$
u_{i c}(t)=f_{i 2}\left(x_{i}(t)\right)^{-1}\left(g_{i}\left(x_{i}(t), \bar{x}_{i}(t)\right)+\Omega_{i}\left(t-t_{0}\right) h_{i}\left(x_{i}(t)\right)\right)
$$

However, since the fault function and interconnection term are unknown, $u_{i c}(t)$ cannot be practically determined this way. Therefore the output of the detection OLA $\hat{h}_{i}\left(x_{i}(t) ; \hat{\theta}_{i}(t)\right)$ which estimates the fault dynamics along with another online approximator referred to as fault accommodation OLA is used to compensate for the interconnection effects. These two OLAs are utilized to construct the estimated $u_{i c}(t)$ as follows

$$
\begin{aligned}
\hat{u}_{i c}(t)= & f_{i 2}\left(x_{i}(t)\right)^{-1}\left(-\hat{q}_{i}\left(\bar{e}_{i}(t) ; \hat{W}_{i}(t)\right)-\hat{h}_{i}\left(x_{i}(t) ; \hat{\theta}_{i}(t)\right)\right) \\
& +f_{i 2}\left(x_{i}(t)\right)^{-1}\left(-l_{i} B_{i}^{T} P_{i} \bar{e}_{i}(t)\right)
\end{aligned}
$$

where $l_{i}$ is a positive constant defined by the user and $P_{i}>0$ is obtained from the Lyapunov equation $P_{i}\left(A_{i}-B_{i} K_{i}^{T}\right)+$
$\left(A_{i}-B_{i} K_{i}^{T}\right)^{T} P_{i}=-Q_{i}$ for any positive definite matrix $Q_{i}$. Moreover $\hat{q}_{i}\left(\bar{e}_{i}(t) ; \widehat{W}_{i}(t)\right)$ is defined as the output of the online approximator from accommodation OLA

$$
\hat{q}_{i}\left(\bar{e}_{i}(t) ; \hat{W}_{i}(t)\right)=\hat{W}_{i}^{T}(t) \psi_{i}\left(\bar{e}_{i}(t)\right)
$$

which is turned on upon detection of a fault, $\psi_{i}\left(\bar{e}_{i}(t)\right)$ is the basis function and $\widehat{W}_{i}$ is the estimated parameter vector which will be updated by an adaptive update law (to be defined later) in order to ensure the stability of the accommodation scheme.

Note that $h_{i}\left(x_{i}(t)\right)=\hat{h}_{i}\left(x_{i}(t) ; \hat{\theta}_{i}(t)\right)=0$ if $i \neq k$. Thus $\tilde{\theta}_{i}^{T}(t) \phi_{i}\left(x_{i}(t)\right)+\varepsilon_{i}(t)=\tilde{h}_{i}=0$ if $i \neq k$. Then the tracking error dynamics can be rewritten as

$$
\begin{aligned}
\dot{\bar{e}}_{i}(t)= & A_{i} \bar{e}_{i}(t)+B_{i}\left[-K_{i}^{T} \bar{e}_{i}(t)-B_{i}^{T} P_{i} \bar{e}_{i}(t)-\hat{W}_{i}^{T}(t) \psi_{i}\left(x_{i}(t)\right)\right. \\
& \left.+\eta_{i}(x(t))+g_{i}\left(x_{i}(t), \bar{x}_{i}(t)\right)+\tilde{\theta}_{i}^{T}(t) \phi_{i}\left(x_{i}(t)\right)+\varepsilon_{i}(t)\right]
\end{aligned}
$$

Furthermore, we define $s_{i}(t)=B_{i}^{T} P_{i} \bar{e}_{i}(t)$ for convenience in the proof of next theorem. Before considering the performance of fault accommodation scheme, Assumption 4 which is another version of Assumption 3 is introduced where the interconnection is represented as a function of tracking errors instead of detection residuals (as in Assumption 3).

Assumption 4: The interconnection terms are unknown but expressed as $\quad g_{i}(x(t)) \leq \varsigma_{i 0}+\sum_{j=1}^{N} \varsigma_{i j}\left(\bar{e}_{j}(t)\right), i=$ $1,2, \ldots, N$, where $\zeta_{i 0}$ for $i=1,2, \ldots, N$ are unknown constants and $S_{i j}($.$) for i, j=1,2, \ldots, N$ are unknown smooth functions. In addition, the interconnection terms can be represented as $g_{i}(x(t)) \leq \zeta_{i 0}+\sum_{j=1}^{N}\left|s_{j}\right| \zeta_{i j}\left(\bar{e}_{j}(t)\right), i=$ $1,2, \ldots, N$ where $\zeta_{i 0}$ are unknown constants and $\zeta_{i j}($.$) are$ smooth functions.

Theorem 2 (Performance of the Fault Accommodation Scheme): Consider the large-scale interconnected system described by (1). Upon detecting a fault in the $k^{\text {th }}$ subsystem, let the control input of all the subsystems be augmented as $u_{i}(t)=u_{i d}(t)+\hat{u}_{i c}(t)$ where $\hat{u}_{i c}(t)$ is defined in (7) and the parameter update law of the accommodation online approximator is given by

$$
\dot{\hat{W}}_{i}(t)=\beta_{i} \psi_{i}\left(\bar{e}_{i}(t)\right) s_{i}(t)-v_{i} \hat{W}_{i}(t)
$$

with $v_{i}$ and $\beta_{i}$ are positive constants, Then the tracking errors $\bar{e}_{i}(t)$, and parameter estimation errors $\widetilde{W}_{i}(t)=W_{i}-\widehat{W}_{i}(t)$ are UUB, if the design parameters are selected such that

$$
l_{i}>\frac{5+N}{2}
$$

Remark 4: Note that the detection OLA approximates the fault function $\hat{h}_{k}$ (with a bounded error), while the accommodation OLA which is generating $\hat{q}_{i}$ is not estimating the interconnection function $g_{i}$, but it is used to approximate $\quad q_{i}\left(\bar{e}_{i}(t)\right)=\frac{s_{i}(t)}{2} \sum_{j=1}^{N} \zeta_{j i}^{2}\left(\bar{e}_{i}(t)\right) . \quad$ In 
otherwords, all the accommodation OLAs must be utilized together to cancel all the interconnection terms.

Figure 1 shows magnitude of the tracking error in the faulty subsystem. The fault will definitely cause the tracking error to increase, since the nominal controller does not have any information about the fault dynamics. After detection of fault, the accommodation scheme will modify the controller to mitigate fault effects and decrease the tracking error.

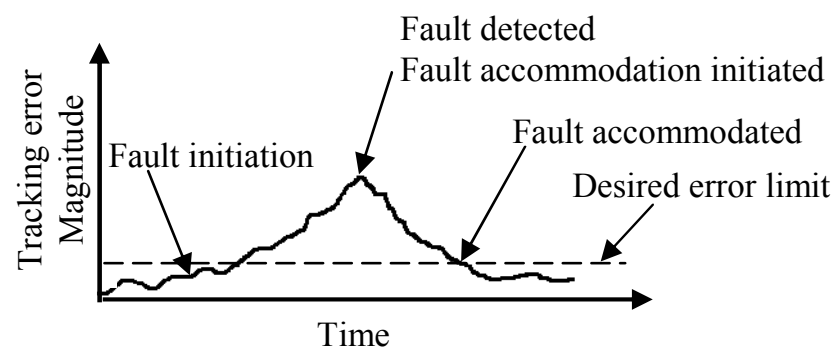

Fig. 1: Tracking error with fault accommodation

\section{Simulation Results}

A system of double inverted pendulums [14], which is depicted in Fig. 2, is used to verify the proposed decentralized detection and accommodation schemes. The pendulums are connected to each other by a spring and their motion dynamics are described by

$\dot{\theta}_{1}=\omega_{1}, \quad \dot{\omega}_{1}=\frac{m_{1} g l_{1} \sin \theta_{1}-b_{1} \omega_{1}}{m_{1} l_{1}^{2}}+\frac{u_{1}}{m_{1} l_{1}^{2}}+\frac{F a_{1} \cos \left(\theta_{1}-\beta\right)}{m_{1} l_{1}^{2}}$

$\dot{\theta}_{2}=\omega_{2}, \quad \dot{\omega}_{2}=\frac{m_{2} g l_{2} \sin \theta_{2}-b_{2} \omega_{2}}{m_{2} l_{2}^{2}}+\frac{u_{2}}{m_{2} l_{2}^{2}}-\frac{F a_{2} \cos \left(\theta_{2}-\beta\right)}{m_{2} l_{2}^{2}}$

where $\theta_{1}=x_{11}$ and $\theta_{2}=x_{21}$ are the angular displacements of the pendulums from the vertical position, $\omega_{1}=x_{12}$ and $\omega_{2}=x_{22}$ are the angular velocities of the pendulums, $b_{1}$ and $b_{2}$ are damping coefficients, $g$ is standard gravity, $m_{1}$ and $m_{2}$ are masses and $l_{1}$ and $l_{2}$ are lengths of the pendulums. Moreover

$$
\begin{gathered}
F=k\left(1+A^{2}\left(l_{k}-l_{0}\right)^{2}\right)\left(l_{k}-l_{0}\right), \beta=\arctan \left(\frac{a_{1} \cos \theta_{1}-a_{2} \cos \theta_{2}}{l_{0}-a_{1} \sin \theta_{1}+a_{2} \sin \theta_{2}}\right) \\
l_{k}=\sqrt{\left(l_{0}+a_{2} \sin \theta_{1}-a_{1} \sin \theta_{1}\right)^{2}+\left(a_{1} \cos \theta_{1}-a_{2} \cos \theta_{2}\right)^{2}}
\end{gathered}
$$

Uncertainties in the form of $\eta_{i}(x(t))=10^{-2} \cos \left(x_{i 1}(t)\right)$ are added to the dynamics of the system and a fault is seeded in the first pendulum subsystem at time $t_{0}=10 \mathrm{sec}$. The fault is described by

$\Omega_{1}\left(t-t_{0}\right) h_{1}\left(x_{1}(t)\right)= \begin{cases}0, & \text { if } t<t_{0} \\ \left(1-e^{-0.1\left(t-t_{0}\right)}\right) \frac{x_{11}(t)+10}{m_{1} l_{1}^{2}}, & \text { if } t \geq t_{0}\end{cases}$

The values of system parameters are provided as $a_{1}=0.1$, $a_{2}=0.35, b_{1}=b_{2}=0.009, m_{1}=0.4, m_{2}=0.5, l_{1}=0.3$, $l_{2}=0.35, l_{0}=0.4, k=30$, and $A=0.1$. The initial states are $\left[\theta_{1}(0), \omega_{1}(0)\right]^{T}=\left[\theta_{2}(0), \omega_{2}(0)\right]^{T}=[0.2,0]^{T}, \quad$ the desired trajectories are $x_{1 d}(t)=\theta_{1 d}(t)=0.55 \cos (3 t)$ and $x_{2 d}(t)=\theta_{2 d}(t)=0.35 \cos (4 t)$ and the simulation runs for 50 seconds. The control inputs in healthy conditions are

$$
\begin{aligned}
& u_{1 d}(t)=-m_{1} g l_{1} \sin x_{11}(t)+b_{1} x_{12}(t)+m_{1} l_{1}^{2}\left(\ddot{x}_{1 d}(t)-K^{T} \bar{e}_{1}(t)\right) \\
& u_{2 d}(t)=-m_{2} g l_{2} \sin x_{21}(t)+b_{2} x_{22}(t)+m_{2} l_{2}^{2}\left(\ddot{x}_{2 d}(t)-K^{T} \bar{e}_{2}(t)\right)
\end{aligned}
$$

where $\bar{e}_{i}$ is the tracking error vector defined by $\bar{e}_{i}=$ $\left[x_{i 1}-x_{i d}, x_{i 2}-\dot{x}_{i d}\right]^{T}$ and $K=[100,1]^{T}$.

To perform the decentralized fault detection, the local fault detectors in (2) are utilized. Upon detection the parameter update law in (3) is used to update the unknown parameter vector such that the online approximator in the first LFD estimates the fault function. The LFD parameters are selected as $\lambda=10, \rho=5, \alpha_{1}=50$, and $\gamma_{1}=0.001$. Also after the detection, decentralized fault accommodation is performed and control inputs are augmented as $u_{i}=u_{i d}+\hat{u}_{i c}$ to cancel the fault effects. $\hat{u}_{i c}(t)$ is calculated by

$$
\begin{aligned}
\hat{u}_{i c}(t)= & m_{i} l_{i}^{2}\left(-\hat{q}_{i}\left(\bar{e}_{i}(t) ; \hat{W}_{i}(t)\right)-\hat{h}_{i}\left(x_{i}(t) ; \hat{\theta}_{i}(t)\right)\right) \\
& +m_{i} l_{i}^{2}\left(-c_{i} B_{i}^{T} P_{i} \bar{e}_{i}(t)\right) \quad i=1,2
\end{aligned}
$$

where $\hat{h}_{i}$ is the output of online approximator in $i^{\text {th }}$ LFD, $\hat{q}_{i}$ is the output of accommodation OLA in $\mathrm{i}^{\text {th }}$ subsystem, $B_{1}=$ $B_{2}=\left[\begin{array}{ll}0 & 1\end{array}\right]^{T}, c_{1}=c_{2}=50$, and $P_{1}$ and $P_{2}$ are obtained from solving the Lyapunov equation $P_{i}\left(A_{i}-B_{i} K_{i}^{T}\right)+$ $\left(A_{i}-B_{i} K_{i}^{T}\right)^{T} P_{i}=-Q_{i}$. By choosing $Q_{1}=Q_{2}=I_{2 \times 2}$ we get $P_{1}=P_{2}=\left[\begin{array}{cc}50.505 & 0.005 \\ 0.005 & 50.505\end{array}\right]$. The unknown parameter vector $\widehat{W}_{i}$ is tuned online using update law in (8) with $\beta_{1}=\beta_{2}=50$ and $v_{1}=v_{2}=0.001$. It should also be mentioned that neural networks with five hidden layer neurons are used as online approximators.

Figure 2 shows the detection residual of the first pendulum (subsystem 1) along with the detection threshold. The residual is below the threshold in the healthy operating conditions. When the fault occurs at $t_{0}=10 \mathrm{sec}$, this residual starts to increase and eventually it exceeds the detection threshold at $t=12.66 \mathrm{sec}$. At this point the fault is detected in the first subsystem, the online approximator is turned on to approximate the fault dynamics, and the parameter update law in (3) is used to update the unknown parameters. As observed in Figure 3, the online approximator is able to estimate the fault function with a small error, after the fault is detected. Because of this approximation property of the OLA, the estimator is able to adapt with the changes in the actual system due to the fault and the detection residual falls below the threshold again.

Figure 4 show the tracking error if no fault accommodation is performed. In this case the fault causes the tracking error to increase. In contrast, when the proposed decentralized fault accommodation strategy is adopted, the estimated fault function and another NN are used to cancel the fault effects. It 
can be observed in Figure 5 that the tracking error of pendulum 1 is brought back close to zero upon detection, when the accommodation scheme is applied.

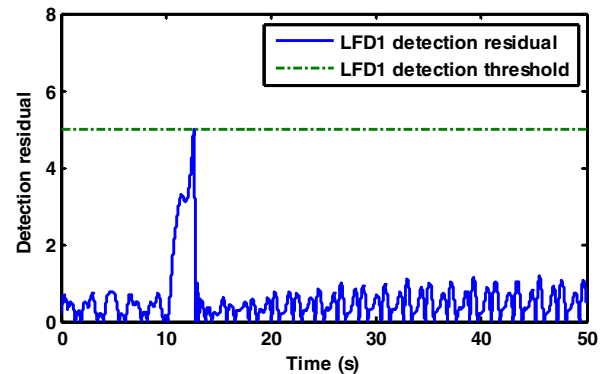

Fig. 2: Subsystem 1 detection residual and threshold

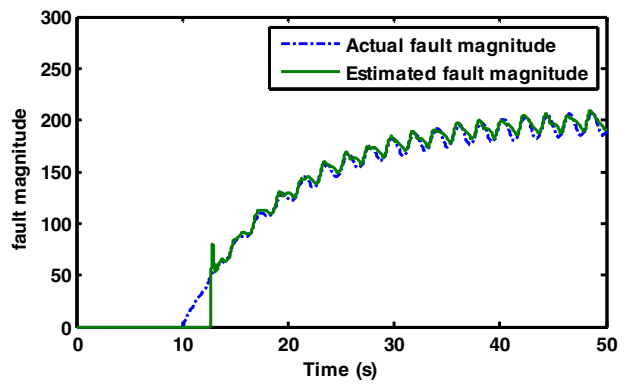

Fig. 3: Actual and estimated fault functions

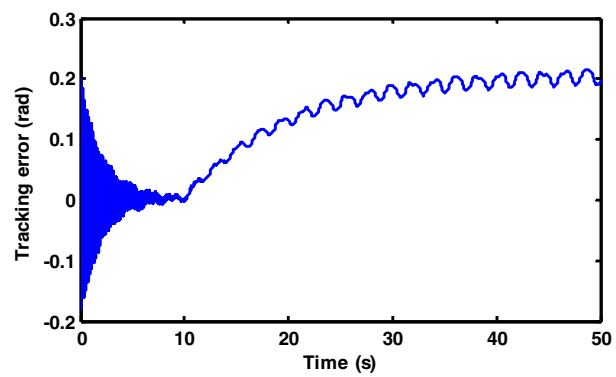

Fig. 4: Pendulum 1 tracking error without accommodation

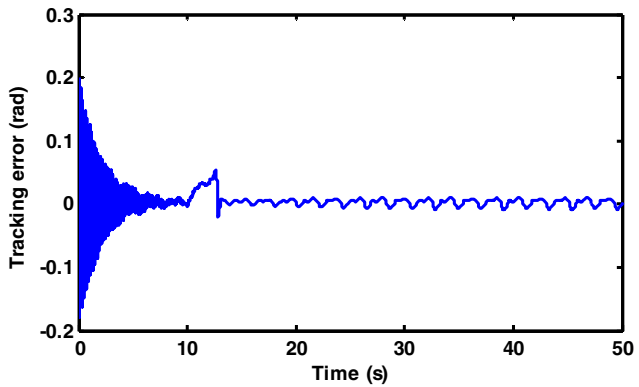

Fig. 5: Pendulum 1 tracking error with accommodation

\section{CONCLUSIONS}

The new decentralized fault detection and accommodation scheme presented in this paper is easy to implement on large-scale industrial systems, where significant amount of communication between subsystems due to state vectors is not possible or desirable. With the proposed scheme, the fault can be detected, its dynamics can be estimated by the detection OLA, and the accommodation can be performed to cancel the effects of the fault on all subsystems without the need for either known interconnection between subsystems, or the measured or estimated non-local state vectors. The future work involves developing a nonlinear estimation method for time-to-accommodation.

\section{REFERENCES}

[1] D. Miljkovic, "Fault detection methods: A literature survey," in MIPRO, 2011 Proceedings of the 34th International Convention, 2011, pp. $750-755$.

[2] H. Hammouri, M. Kinnaert, and E. H. El Yaagoubi, "Observer-based approach to fault detection and isolation for nonlinear systems," Automatic Control, IEEE Transactions on, vol. 44, pp. 1879-1884, 1999.

[3] R. J. Patton, J. Chen, and T. M. Siew, "Fault diagnosis in nonlinear dynamic systems via neural networks," in Control, 1994. Control '94. International Conference on, 1994, pp. 1346-1351 vol.2.

[4] C. De Persis and A. Isidori, "A geometric approach to nonlinear fault detection and isolation," Automatic Control, IEEE Transactions on, vol. 46, pp. 853-865, 2001

[5] S. N. Huang and T. Kok Kiang, "Fault Detection, Isolation, and Accommodation Control in Robotic Systems," Automation Science and Engineering, IEEE Transactions on, vol. 5, pp. 480-489, 2008.

[6] J. Bin, M. Staroswiecki, and V. Cocquempot, "Fault Accommodation for Nonlinear Dynamic Systems," Automatic Control, IEEE Transactions on, vol. 51, pp. 1578-1583, 2006.

[7] N. Meskin and K. Khorasani, "Fault Detection and Isolation of Distributed Time-Delay Systems," Automatic Control, IEEE Transactions on, vol. 54, pp. 2680-2685, 2009.

[8] Z. Yingwei, Z. Hong, S. J. Qin, and C. Tianyou, "Decentralized Fault Diagnosis of Large-Scale Processes Using Multiblock Kernel Partial Least Squares," Industrial Informatics, IEEE Transactions on, vol. 6 , pp. 3-10, 2010

[9] L. Peng, Z. Bo, and L. Yuanchun, "Fault detection methods for reconfigurable manipulators via decentralized adaptive fuzzy nonlinear observer," in Industrial Engineering and Engineering Management (IEEM), 2010 IEEE International Conference on, 2010, pp. 1937-1941.

[10] M. A. Demetriou, K. Ito, and R. C. Smith, "Adaptive Monitoring and Accommodation of Nonlinear Actuator Faults in Positive Real Infinite Dimensional Systems," Automatic Control, IEEE Transactions on, vol. 52, pp. 2332-2338, 2007

[11] P. Panagi and M. M. Polycarpou, "Distributed Fault Accommodation for a Class of Interconnected Nonlinear Systems With Partial Communication," Automatic Control, IEEE Transactions on, vol. 56, pp. 2962-2967, 2011.

[12] J. D. Boskovic and R. K. Mehra, "A decentralized scheme for accommodation of multiple simultaneous actuator failures," in American Control Conference, 2002. Proceedings of the 2002, 2002, pp. 5098-5103 vol.6.

[13] S. Jagannathan, Neural Network Control of Nonlinear Discrete -time Systems. NY: CRC publications, 2006.

[14] H. Sunan, T. Kok Kiong, and L. Tong Heng, "Decentralized control design for large-scale systems with strong interconnections using neural networks," Automatic Control, IEEE Transactions on, vol. 48, pp. 805-810, 2003.

[15] H. Ferdowsi, D. L. Raja, and S. Jagannathan, "A decentralized fault detection and prediction scheme for nonlinear interconnected continuous-time systems," in Neural Networks (IJCNN), The 2012 International Joint Conference on, 2012, pp. 1-7.

[16] Y. Qinmin, J. B. Vance, and S. Jagannathan, "Control of Nonaffine Nonlinear Discrete-Time Systems Using Reinforcement-Learning-Based Linearly Parameterized Neural Networks," Systems, Man, and Cybernetics, Part B: Cybernetics, IEEE Transactions on, vol. 38, pp. 994-1001, 2008. 